\section{Diffuse replacement of pancreatic parenchyma by intraductal papillary mucinous neoplasm}

A 63-year-old insulin-dependent diabetic woman presented to our multidisciplinary group for evaluation and management of multifocal cystic lesions of the pancreas found incidentally by computed tomography during an evaluation of diverticulitis. Initial assessment included endoscopic ultrasound (EUS) with fine-needle aspiration (FNA) by curved linear array echoendoscope. Near-total replacement of the pancreatic parenchyma by innu- merable cysts of various size and character was demonstrated, involving all regions of the pancreas ( $\bullet$ Fig. 1 ; Video 1 ). Several cysts exhibited features that gave cause for concern, including size well over $30 \mathrm{~mm}$, thickened walls, and thickened septations, as well as round, mobile solid structures suggestive of mucin balls ( Fig.2a). Moreover, the main pancreatic duct appeared to communicate with the cysts in the regions of the head and neck
(๑ Video 1). The minimally spared parenchyma was heterogeneous without lobularity. The major vessels of the abdomen were unremarkable and without abnormality, nor was there evidence of other regional disease or lymphadenopathy. FNA was performed to recover fluid contents from a larger cystic component, and a second pass was performed to confirm the mobility and benign nature of a suspected mucin ball ( Fig. 2 b).

\section{Video 1}

Endoscopic ultrasound demonstrating nearcomplete replacement of the entire pancreatic parenchyma.
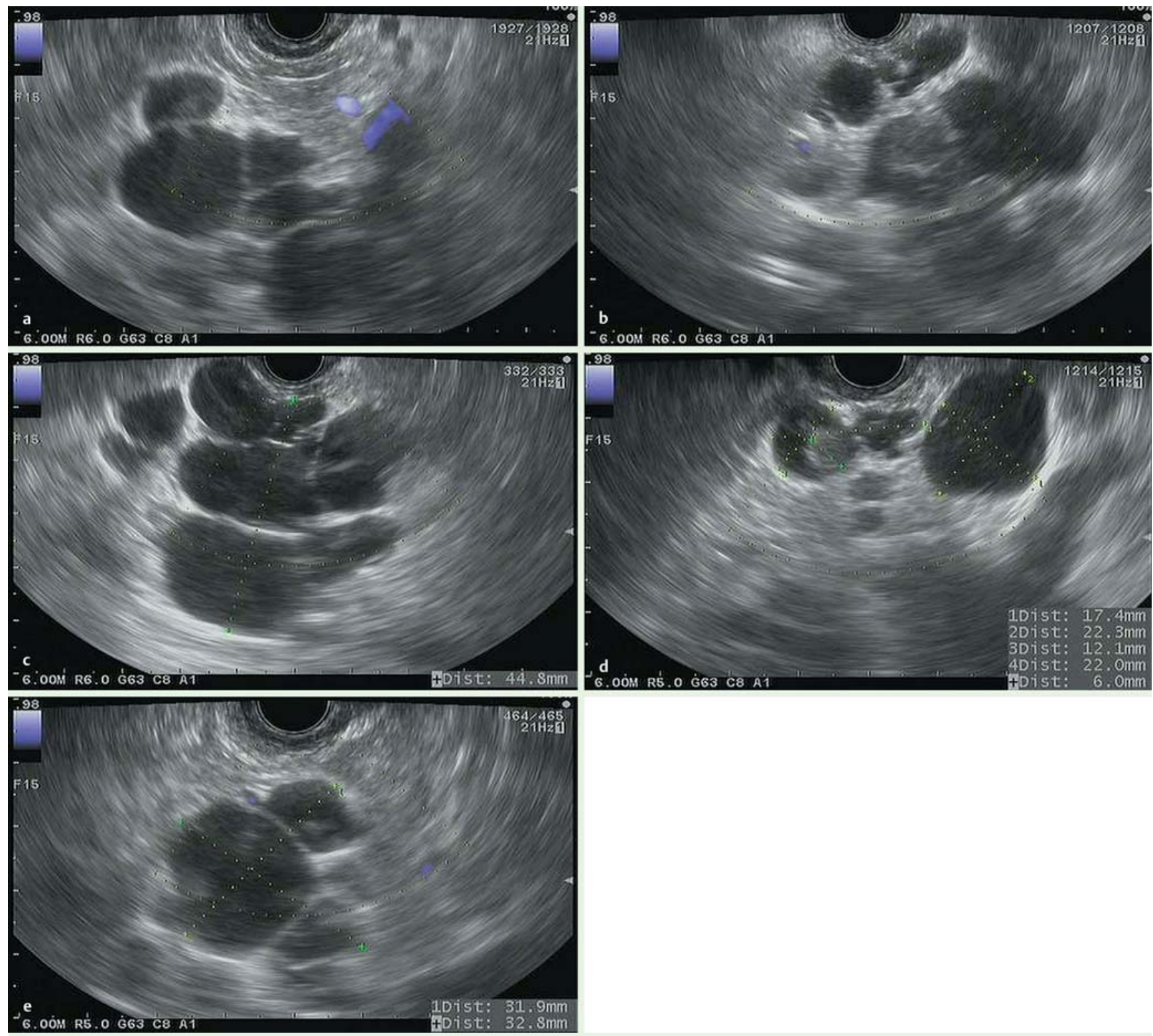

Fig. 1 a-e Endoscopic images demonstrating near-complete replacement of the pancreatic parenchyma: a uncinate process and head, $\mathbf{b}$ head, $\mathbf{c}$ neck, d body, e tail. 


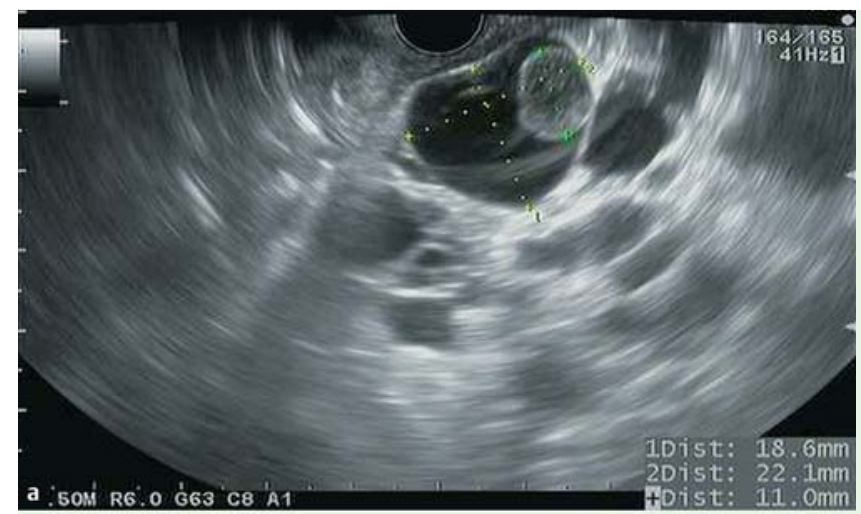

Fig. 2 a Well-defined round structure within a larger cyst; on fineneedle aspiration it was found to be mobile $\mathbf{b}$.
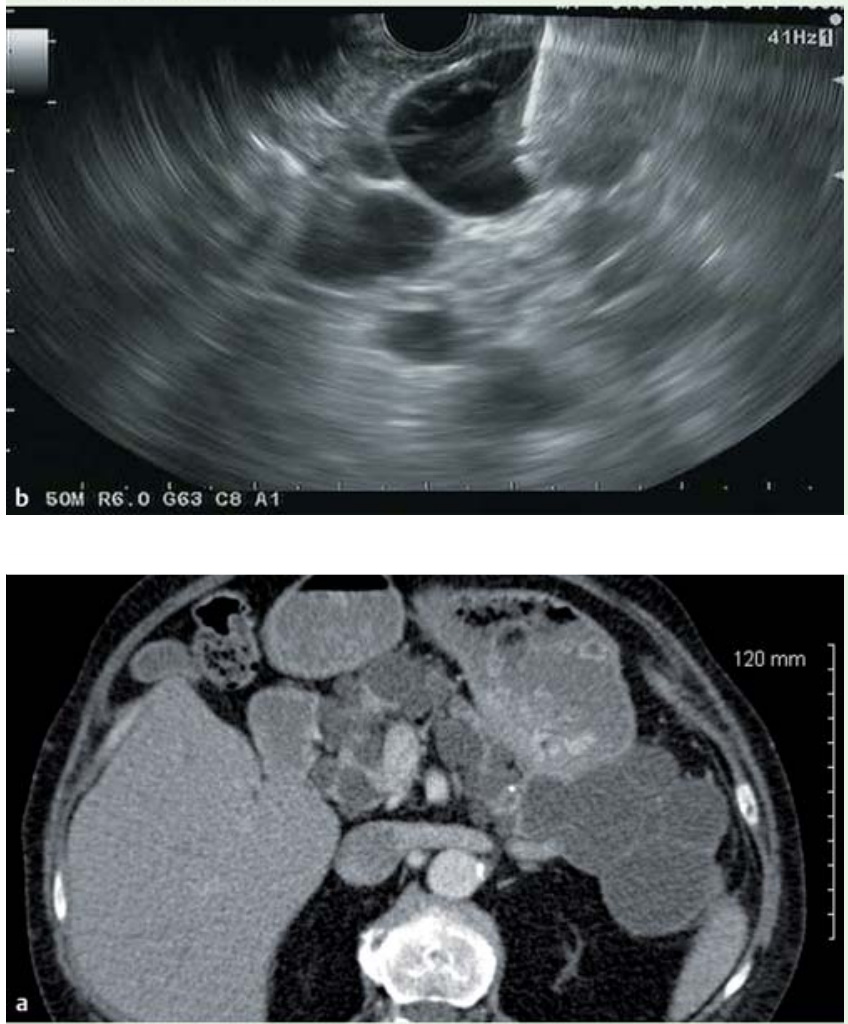

Fig. 3 a, b Contrastenhanced computed tomography confirming near complete replacement by cystic lesions: a transverse and $\mathbf{b}$ coronal views.

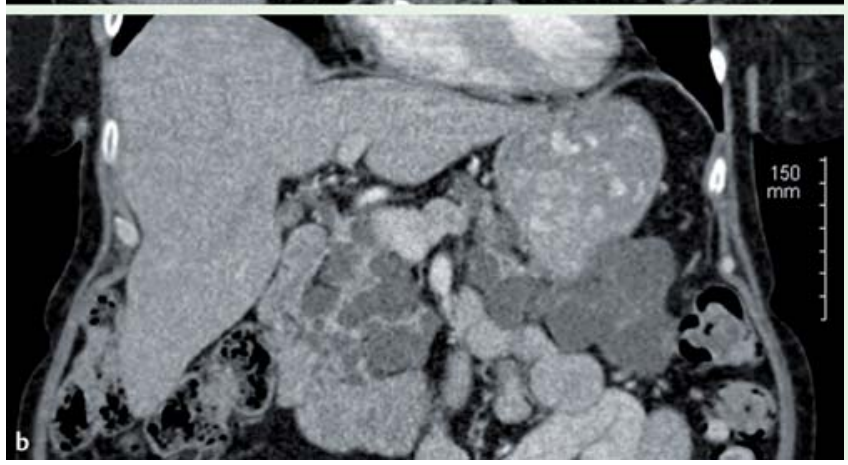

Fluid analysis returned with relatively high levels of both amylase (26900U/L) and carcinoembryonic antigen (CEA; $589 \mathrm{ng} / \mathrm{mL}$ ), and associated cytopathology was paucicellular without suspicious cells, although a thick mucus component was noted. Serum tumor marker evaluation included a cancer antigen (CA) 19-9 concentration of $1739 \mathrm{U} / \mathrm{mL}$ and a CEA concentration of $3.4 \mathrm{ng} / \mathrm{mL}$. Interval pancreat- firmed complete parenchymal replacement by cysts lined by uniform, bland columnar epithelium with abundant mucinous cytoplasm and occasional small micropapillary projections. Interposed between the cysts were variably thick fibrous septae containing focal calcifications, trapped islets, and foci of atrophic pancreatic parenchyma ( Fig.5). The finding of multiple intraductal mucinous cysts is consistent with a diagnosis of diffuse side-branch intraductal papillary mucinous neoplasm (IPMN). The surgical procedure was uncomplicated, and the patient recovered and was discharged with follow-up care as anticipated.

This case presents a unique, previously unreported demonstration of near-complete pancreatic replacement by diffuse side-branch IPMN diagnosed by EUS and confirmed with surgical excision. Accurate differentiation between various etiologies of cystic pancreatic lesions presents challenges even in the most typical of scenarios [1]. By recognizing sometimes subtle distinguishing characteristics, however, the differential diagnosis may frequently be narrowed to one solitary etiology. Here, EUS alone predicted the diagnosis in part by allowing fluid analysis. The study demonstrated a multifocal distribution of thickly septated cysts clearly communicating with the main pancreatic duct, which when aspirated showed a viscous clear fluid rich in CEA and amylase in an elderly patient. While not definitively exclusive, this pattern differs from those expected for mucinous cystic neoplasms (body/tail of pancreas, solitary, multiloculated, ovarian-like stroma, without ductal communication, low amylase), serous cystadenomas (head of the pancreas, solitary, microcystic/sponge-like, central fibrous scar sometimes calcified, without ductal communication, low amylase and CEA), pseudocysts (single, unilocular, patient with history of pancreatitis, low CEA), solid-pseudopapillary neoplasms (body/tail, young female, solid and cystic, without ductal communication, low amylase and CEA), lymphoepithelial cysts (elderly male, cystic, associated with keratinous debris, without ductal communication, low amylase and CEA) and cystic neuroendocrine tumors (nonseptated, without ductal communication, low amylase and CEA) [2]. Given the diffuse nature of the cystic lesions, von Hippel-Lindau disease was also considered; however, this syndrome is associated with serous cystadenomas and/or neuroendocrine lesions, not mucinous neoplasms, as demonstrat- 


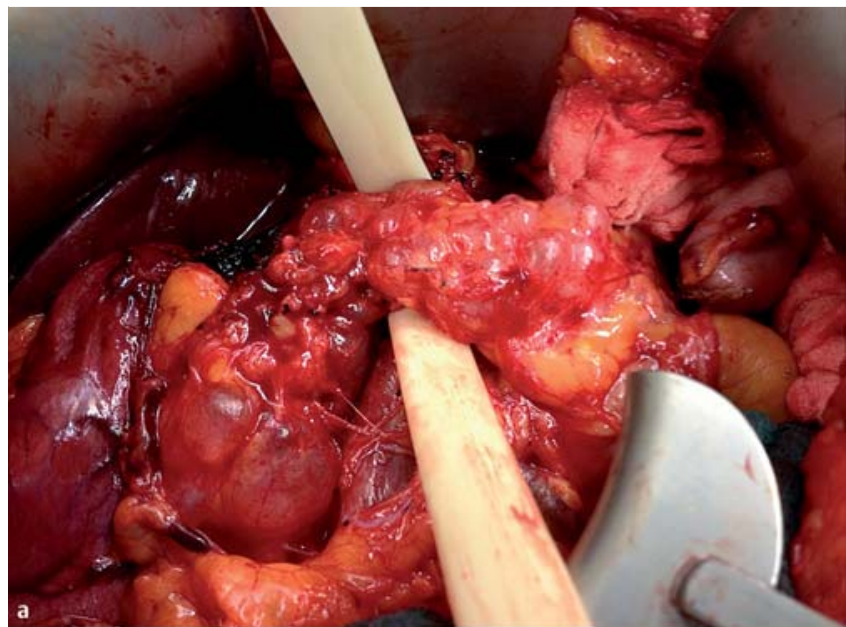

Fig. 4 a, b Gross surgical specimen demonstrating exophytic cystic parenchyma: a in vivo and $\mathbf{b}$ following total pancreatectomy and splenectomy.
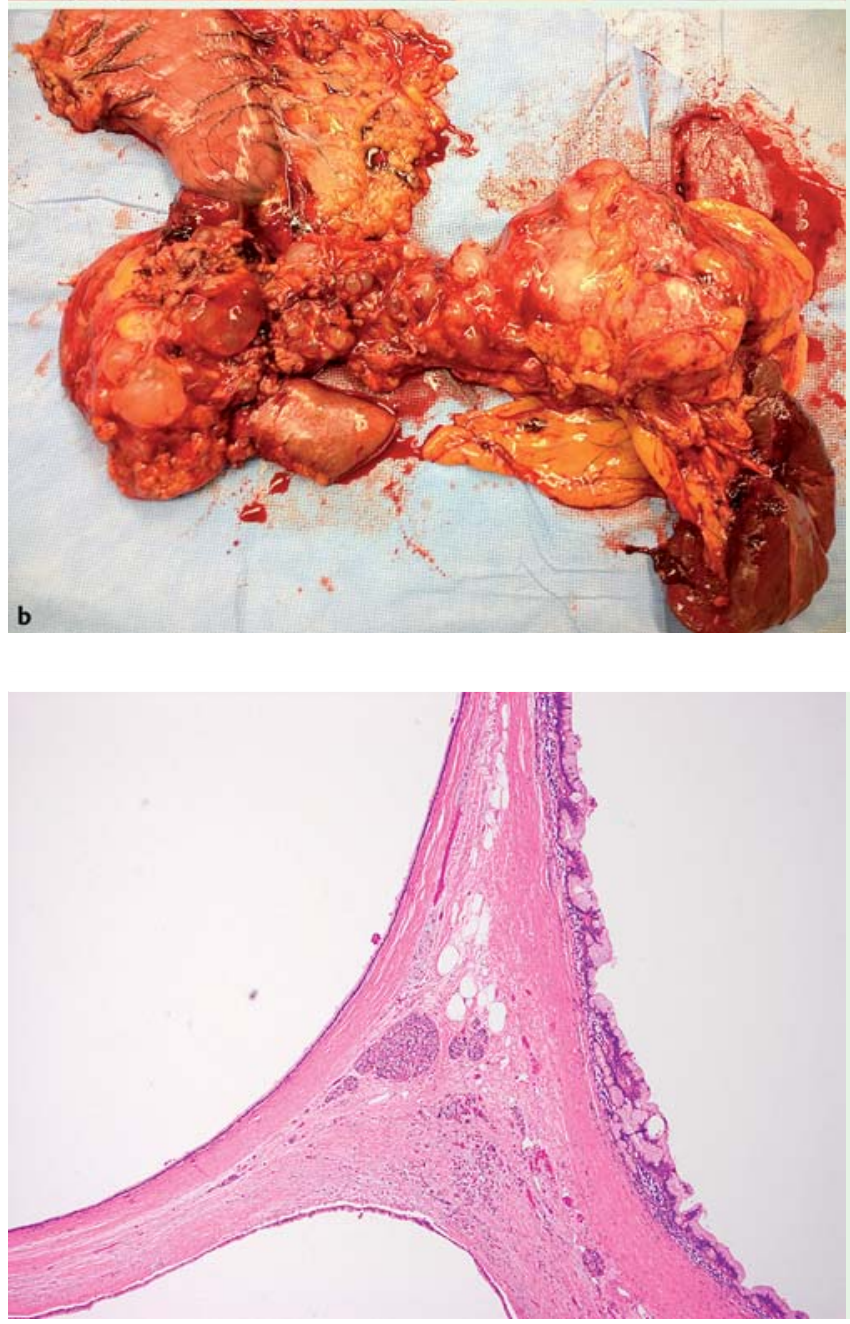

Fig. 5 Representative histology of a cyst with the characteristic findings of an intraductal papillary mucinous neoplasm.

ed by the cytopathology obtained on FNA [3]. Current consensus guidelines for management of IPMNs dictate consideration of total pancreatectomy to address the dif-
Endoscopy_UCTN_Code_CCL_1AF_2AZ_3AB

\section{Competing interests: None}

\section{Stuart K. Amateau ${ }^{1}$, Martine C. McManus $^{2}$, Barish H. Edil ${ }^{3}$}

${ }^{1}$ Department of Internal Medicine, Division of Gastroenterology and Hepatology, University of Colorado Medical Campus, Aurora, Colorado, USA

2 Department of Pathology, University of Colorado Medical Campus, Aurora, Colorado, USA

${ }^{3}$ Department of Surgery, University of Colorado Medical Campus, Aurora, Colorado, USA

\section{References}

1 Yoon WJ, Brugge WR. Pancreatic cystic neoplasms: diagnosis and management. Gastroenterol Clin North Am 2012; 41: 103 118

2 Cunningham SC, Hruban RH, Schulick RD. Differentiating intraductal papillary mucinous neoplasms from other pancreatic cystic lesions. World J Gastrointest Surg 2010; 2: $331-336$

3 Hammel PR, Vilgrain V, Terris B et al. Pancreatic involvement in von Hippel-Lindau disease. The Groupe Francophone d'Etude de la Maladie de von Hippel-Lindau. Gastroenterology 2000; 119: 1087-1095

4 Tanaka M, Fernandez-del Castillo C, Adsay V et al. International consensus guidelines 2012 for the management of IPMN and MCN of the pancreas. Pancreatology 2012; 12: $183-197$

\section{Bibliography}

DOI http://dx.doi.org/

10.1055/s-0033-1344993

Endoscopy 2013; 45: E407-E409

(c) Georg Thieme Verlag KG

Stuttgart · New York

ISSN 0013-726X

\section{Corresponding author}

\section{Stuart K. Amateau, MD, PhD}

Division of Gastroenterology and Hepatology Department of Medicine

University of Colorado Medical Campus MS B-158 AO1 Bldg, Room 7601

12631 East 17th Ave

Denver

CO 80045

USA

Fax: +1-303-724-1891

stuart.amateau@ucdenver.edu 